\title{
How to measure homologous recombination deficiency in ovarian cancer
}

\author{
Claudia Marchetti ${ }^{1}$, Iain A. McNeish ${ }^{2}$
}

\begin{abstract}
Defective DNA repair via homologous recombination (HR) is common in ovarian high grade serous carcinomas, and homologous recombination deficiency (HRD) represents an important therapeutic target in epithelial ovarian cancers (EOCS). The development of poly(ADP ribose) polymerase (PARP) inhibitors (PARPi) has been an important advance in the treatment of HR-deficient EOCs with the potential to change daily clinical practice. However, while germline and somatic mutations in BRCA1 and BRCA2 are still the most important mechanisms of HRD, alterations in other DNA repair pathways might also contribute to defective HR. In this review, we focus on current and emerging approaches for identifying and targeting HR-deficient EOCs, and discuss the challenges associated with these approaches.
\end{abstract}

Key words: HRD assays, ovarian cancer, molecular biology, PARP inhibitors

\section{Introduction}

Genomic and functional studies suggest that approximately $50 \%$ of ovarian high grade serous carcinoma (HGSCs) may exhibit defective DNA repair via homologous recombination (HR) at the time of diagnosis due to genetic and epigenetic alterations of HR pathway genes [1, 2]. Defective HR represents an important therapeutic target in HGSC as shown by the use of poly(ADP ribose) polymerase (PARP) inhibitors (PARPi), which display synthetic lethality when applied to HR-deficient cells [3, 4]. Here, we focus on current and emerging approaches for identifying and targeting HR-deficient epithelial ovarian cancers (EOCs), and discuss challenges associated with these approaches.

\section{HGSC biology: molecular pathogenesis of epithelial ovarian cancer}

Five distinct subtypes of ovarian cancer have been identified: high grade serous, low grade serous, clear cell, endometrioid and mucinous [5]; HGSC is by far the commonest subtype. HGSC is marked by universal mutation of the tumour suppressor gene TP53 [6], as well as high levels of genomic instability [1]. Half of all HGSC carcinomas are estimated to have HR deficiency (HRD) at the time of diagnosis, with about 15\% harbouring a germline mutation in $B R C A 1 / 2,6 \%$ a somatic $B R C A 1 / 2$ mutation, and $20 \%$ a mutation in, or epigenetic silencing of, another HR gene [1,7]. Epigenetic silencing of BRCA1 via promoter hypermethylation occurs in approximately $10-20 \%$ of HGSCs and is mutual- ly exclusive of $B R C A 1$ mutation; this implies that $B R C A$ genes are frequently inactivated by either mutation or epigenetic silencing in EOC [1]. Other HR variations are infrequent $(<2 \%)$, but might involve mutations in Fanconi anaemia (FA) genes (PALB2, FANCA, FANCI, $F A N C L$ and $F A N C C$ ), in several genes of the RAD family ( $R A D 50, R A D 51, R A D 51 C$ and $R A D 54 L$ etc.) and in other genes involved in HR pathway, including $A T M$, ATR, CHEK 1 and CHEK2.

Defective HR in EOC may also occur with alterations of other genes which are known to modulate the HR pathway and indirectly cause HR deficiency. For example, amplification and overexpression of EMSY, reported in 17\% of HGSC [8], is another potential mechanism of HR deficiency. EMSY interacts with the transactivation domain of BRCA2 leading to inhibition of its transcriptional activity. It also co-localizes with BRCA2 at DNA damage sites and interacts with several chromatin remodelling proteins. The

'Department of Obstetrical-Gynecological Sciences and Urological Sciences, "Sapienza" University of Rome, Rome, Italy. Institute of Cancer Sciences, University of Glasgow, Glasgow, UK. Correspondence to:

Claudia Marchetti,

Department of Obstetrics and Gynecology,

"Sapienza" University of Rome,

Viale del Policlinico 155, 00161 Roma, Italy.

Phone: + 393384652744 - Fax: + 390649972564

E-mail: clamarchetti@libero.it

CANCER BREAKING NEWS 2017;5(1):15-20

DOI: $10.19156 / c b n .2017 .0034$ 
correlation between inactivating mutations of $C D K 12$ and HR deficiency has been elucidated [9]. $C D K 12$ is one of the most significantly mutated genes in EOC $(3 \%$ of cases in the TCGA dataset) and is involved in the transcription of $B R C A 1$ and other HR genes; thus, when its activity is reduced, $B R C A 1$ expression is reduced as well as DNA repair by homologous recombination, and this finally leads to an increase of PARPi sensitivity [10].

Importantly, it should be highlighted that other underlying and less clear mechanisms might provoke defective HR in HGSC. Furthermore, variations in the nucleotide excision repair (NER) pathway have been found in up to $8 \%$ high grade serous EOCs as well as alterations of the mismatch repair (MMR) mechanism, which may be damaged in up to $3 \%$ of HGSCs [11].

\section{DNA double strand break repair}

The recognition and subsequent repair of DNA damage is necessary for normal cellular function and genomic stability. It has been demonstrated that acquired or inherited defects in DNA repair pathways result in an increased lifetime risk of cancer [12]. Several pathways have been identified for DNA repair, and they are engaged variably to repair single- (SSB) and double-strand (DSB) DNA breaks. In particular, three mechanisms are involved in DSB repair: nonhomologous end joining (NHEJ) repair, HR repair (HRR) and microhomology-mediated NHEJ (MMEJ), also termed alternative NHEJ (alt-NHEJ). MMEJ is different from the other repair pathways as it requires a 5-25 base pair microhomologous sequences to align the broken strands before joining [13].

These mechanisms are complex and require the accurate temporal and spatial synchronization of a numerous proteins which are involved in both DNA repair processes and in cell cycle control, until the repair is complete. HRR is an error-free pathway that uses a homologous DNA template to repair DSB and is initiated by end resection of the DNA ends to generate a long stretch of single-strand DNA for strand invasion. HRR can only be utilised when cells enter S and G2 because cyclin-dependent kinases are needed for promoting end resection and to activate HRR, and a homologous donor strand is available. The majority of DSB arise during DNA replication when a replication fork encounters an unrepaired SSB; the HRR pathway, together with the nuclear enzyme, PARP-1, is particularly important for repairing these collapsed replication forks [3, 14]. Double-strand DNA breaks are recognized by ATM kinase, which phosphorylates downstream targets including CHEK2, p53, BRCA1 and H2AX. BRCA1, assisted by BARD1 and BRIP1, acts as a scaffold that organizes the remaining proteins to the site of repair. The MRN complex, which consists of MRE11, RAD50 and NBS1, then resects the DNA to form 3' overhangs that are bound by RPA. BRCA2 is recruited with the assistance of PALB2 and loads RAD51 onto RPA-coated DNA with the assistance of RAD51B, RAD51C and RAD51D. The RAD51 nucleoprotein filament then invades the homologous DNA strand in a process called strand invasion, allowing the remaining DNA repair to occur with the use of the sister chromatid as a template for error-free repair [15]. Thus, BRCA1 and BRCA2 are crucial to the accurate execution of HR repair.

\section{Assays of HR function}

Multiple studies have demonstrated that tumours with loss of BRCA1/2 function have a higher sensitivity to platinum compounds and PARPi [16-18]. However, many HGSC that do not have a mutation in $B R C A 1 / 2$ or another HR gene show $B R C A$ mutant-like behaviour [10, 19]. Therefore, defining those tumours with defective HR might help in the clinical management of these patients. Indeed, in order to offer PARPi to all HGSC patients with defective HR, considerable efforts have been made to identify somatic signatures of abnormal HR. Although there are gold-standard laboratory assays of HR function (e.g. DR-GFP [20] and RAD51 [2] focus formation assays), these are not suitable for routine use in clinical samples. In particular these functional HR deficiency assays demand a live tumour sample that can be grown for several days, which precludes the use of fixed or paraffin embedded specimens. There are three other approaches that may be of clinical use: response to platinum chemotherapy, genomic scarring and next generation sequencing (NGS) of HR genes.

Loss of BRCA1/2 function is associated with response to platinum chemotherapy. In single agent olaparib studies, response to PARP inhibition was correlated with platinum sensitivity, with more frequent and durable responses in tumours that had relapsed in the platinum-sensitive timeframe ( $>6$ months following last platinum exposure) [21]. Similarly, Ledermann et al. showed clearly that maintenance olaparib produced a highly significant progressionfree survival (PFS) advantage compared to placebo when administered to women with relapsed HGSC who had responded to platinum chemotherapy in the relapse setting, independently of $B R C A$ mutation status [22]. Thus, at a first approximation, true platinum sensitivity is able to identify patients who may benefit from PARP inhibition. However, this is only applicable for maintenance treatment where prior platinum response has been demonstrated, and will not be applicable in the first-line set- 
ting where patients have undergone complete debulking and have no visible disease to allow platinum response to be assessed. Moreover, in the treatment setting, Gelmon et al. demonstrated robust responses in some patients with relapsed HGSC who were $B R C A$ wild-type and who had relapsed in the platinum-resistant timeframe [23], indicating that the simple clinical criteria of 'platinumsensitive' and 'platinum-resistant' relapse are inadequate. Thus, there is a need to develop robust assays that can assess HR status.

Ovarian HGSC exhibits a high burden of genomic aberration. Mainly, there are two important types of aberrations: (1) regional copy number aberrations (CNAs) and (2) structural rearrangements. CNAs are described as loss or gain in the number of delineated, subchromosomal section of DNA copies. Whereas structural rearrangements are defined as changes into precise location or orientation of known DNA sequence. Structural rearrangements include DNA recombinations (material exchange between homologous regions), DNA translocations (exchange of material between non-homologous regions) and DNA inversions (change into defined sequence orientation) [24].

The potential outcome of DNA recombinations is the regional loss of heterozygosity ( $\mathrm{LOH}$ ), in which one parental copy of heterozygous DNA region is lost and the other is preserved. This leads to an imbalance in the ratio of parental alleles from the normal 1:1 and a cell exhibits a 'structural chromosomal instability' [25].

Three independent DNA-based measures of genomic instability have been developed, based on LOH [26], telomeric allelic imbalance (TAI) [27], and large-scale state transitions (LST, a measure of the number of chromosomal breaks between adjacent regions of $\geq 10 \mathrm{Mb}$ ) [28]. Importantly, the sum of the three scores was better in discriminating HR deficient from non-deficient tumours. There was also a linkage between genome signature to platinum sensitivity [27] and/or BRCA1 inactivation [22-24], and a significant correlation between the three scores was identified [29].

On this basis, the Myriad myChoice HRD has been developed [6], which uses an unweighted sum of the three independent DNA-based measures of genomic instability above mentioned in order to indentify an HRD score [30]. The relationship between response to therapy and the presence of germline $B R C A$ mutation ( $\mathrm{gBRCAm}$ ) or HRD score (defined as HRD score $\geq 42$ ) has been confirmed in three studies focused on neoadjuvant platinum containing therapy in triple-negative breast cancer patients [30].

A second assay has been developed by Foundation Medi- cine, using formalin-fixed paraffin-embedded (FFPE) tissue. The NGS assay combines somatic BRCA status as well as the percentage of genome-wide $\mathrm{LOH}$, quantified in approximately 3500 single-nucleotide polymorphisms (SNPs) throughout the genome, to define these subgroups: $B R C A$ mutant (deleterious germline or somatic), $B R C A \mathrm{WT} / \mathrm{LOH}$ high or BRCA WT/LOH low. Foundation has partnered with Clovis Oncology to develop a companion diagnostics (CDx) in parallel with the clinical development of rucaparib in the ARIEL2 and 3 studies (NCT01968213) (see below).

However, it has been suggested [10] that these assays might have shortcomings as they are not able to detect the reversal of HR deficiency that may appear when cell become resistant to platinum and PARPi, for example through acquisition of secondary revertant mutations that restore BRCA1/2 function, which have been detected in both cell lines and tumour samples [31, 32].

Beyond genome scars and functional tests, other strategies are currently under investigation, including gene-expression profile (GEP) signatures and protein signatures of "HRness" within tumours. Interestingly, Konstantinopoulos developed a 60-gene expression signature of $B R C A$ ness in sporadic or germline $B R C A$-associated EOC [33]. This signature was able to predict platinum responsiveness in 8 out of 10 patient-derived tumour specimens and was then validated in 70 patients with sporadic disease: it was found that patients with the $B R C A$-like profile had an increase in both disease-free survival and overall survival (OS) compared with patients with a non- $B R C A$-like profile. Further studies are needed to confirm these findings and also to evaluate whether these gene expression signatures are truly predictive of response to therapy rather than being only prognostic.

More recently, based on the fact that abrogation of BRCA1 and BRCA2 function leads to a characteristic set of mutational signatures, a new HRD assay (HRDetect) has been developed which was able to identify six distinguishing mutational signatures predictive of BRCA1/ $B R C A 2$ deficiency [34]. In the paper from Davies et al., it was found that integrating all of the classes of mutational signatures reveals a larger proportion of individuals harbouring $B R C A 1 / B R C A 2$ deficiency. In fact, although only 22 patients were originally recruited with known germline BRCA1- or BRCA2-null cancers, HRDetect revealed an additional 33 tumours with a germline mutation, 22 tumours with a somatic mutation bringing the total number of BRCA1/BRCA2-deficient tumours to 124 (22\%). Largescale population-based studies as well as application in clinical trials are required to gather proper population estimates. 


\section{Clinical trial data}

Several trials have been conducted with the aim of identifying HR deficient patients who might benefit from PARP inhibition. In addition to the results obtained with olaparib mentioned above, data from two other PARPi are now available and have the potential to change our daily practice.

Niraparib (MK-4827) is a selective PARP-1 and -2 inhibitor (PARP1/2 Ki 3.2/4.0 nM) [35] which has been investigated in both germline $B R C A$ mutated $(\mathrm{gBRCAm})$ and $B R C A$ wild-type EOC $[36,37]$. In October 2016, the results of the phase III ENGOT (European Network for Gynecological Oncological Trial groups)-OV16/NOVA trial were published [17]. NOVA examined the role of niraparib as maintenance treatment following response to platinum-based chemotherapy in patients with relapsed HGSC. Patients were stratified based on germline $B R C A$ mutation status, and randomised 2:1 to receive niraparib or placebo. In order to identify $B R C A$ wild-type patients who might benefit from niraparib, the myChoice HRD assay [6] was applied to archival tumour tissue samples, and used to classify the population of patients in the gBRCAm wild-type cohort as being either HRD positive or negative. The trial enrolled 553 patients, with 203 in the gBRCAm group (138 allocated to oral niraparib $300 \mathrm{mg}$ and 65 to placebo) and 350 patients in the non-gBRCAm cohort (234 allocated to oral niraparib $300 \mathrm{mg}$ and 116 to placebo). The study met its primary endpoint with a significant improvement of PFS compared with placebo. Median PFS with niraparib compared to placebo was 21.0 versus 5.5 months (hazard ratio [HR] $0.27 ; \mathrm{p}<0.0001$ ) in the $g B R C A m$ group ( $\mathrm{n}=203) ; 9.3$ versus 3.9 months (HR $0.45 ; \mathrm{p}<0.001)$ in the non-germline $B R C A$ mutated group $(\mathrm{n}=350)$. Median PFS in the non- $g B R C A$ cohort was 12.9 versus 3.8 months (HR $0.38 ; \mathrm{p}<0.001)$ in the subgroup who were classified as HRD positive, and 6.9 versus 3.8 months (HR 0.58; $\mathrm{p}=0.0226)$ in HRD-negative $(\mathrm{n}=134)$ patients.

Compared to placebo, niraparib also significantly prolonged the second PFS, time to first subsequent treatment, and chemotherapy-free interval in the mutation and mutation-free groups, as well as in the HRD subgroup.

In March 2017, the U.S. Food and Drug Administration approved niraparib for the maintenance treatment of recurrent epithelial ovarian, fallopian tube or primary peritoneal cancer, who have had complete or partial response to the last platinum-based chemotherapy, regardless of $B R C A$ or HRD status [38].

The first PARPi to be administered in humans was rucaparib. Two important trials are currently ongoing for rucaparib in ovarian cancer: the phase II and phase III
ARIEL2 and ARIEL3 (NCT01891344 and NCT01968213, respectively). ARIEL2 is a two-part study; part 1 has been completed and part 2 is currently enrolling. Part 1 investigated oral rucaparib as single agent treatment in recurrent platinum-sensitive high-grade epithelial ovarian, primary peritoneal, or fallopian tube cancer [18]. As previously mentioned, it also assessed the capability of an integrated Foundation Medicine HRD assay to predict response to rucaparib by the prospectively defining three subgroups, defined above.

Patients were subdivided into three groups $B R C A$ mutant (deleterious germline or somatic), $B R C A \mathrm{WT} / \mathrm{LOH}$ high ( $\mathrm{LOH} \geq 14 \%$ ), or $B R C A \mathrm{WT} / \mathrm{LOH}$ low ( $\mathrm{LOH}<14 \%)$. The primary endpoint was PFS, which was longer in rucaparib-treated patients with $B R C A$ mutations (12.8 months; 95\% confidence interval [CI] 9.0-14.7) or $B R C A \mathrm{WT} /$ LOH high platinum-sensitive disease (5.7 months; $95 \% \mathrm{CI}$ 5.3-7.6) than in BRCA WT/LOH low carcinomas (5.2 months; 95\% CI 3.6-5.5; $\mathrm{p}<0.0001$ for $B R C A$ mutant vs $B R C A$ WT/LOH high; $\mathrm{p}=0.011$ for $B R C A$ mutant $v s$ $B R C A \mathrm{WT} / \mathrm{LOH}$ low).

It was also found that the proportion of patients who achieved a response (objective response by combined Response Evaluation Criteria in Solid Tumors [RECIST] and cancer antigen [CA]125) was similar irrespective of whether the $B R C A$ mutation was germline $(85 \%)$ or somatic (84\%) or whether a patient had a BRCA1 (86\%) or $B R C A 2(82 \%)$ mutation. Moreover, all 4 patients with a mutation in RAD51C were classified as $\mathrm{LOH}$ high, and 3 of these 4 showed a RECIST response, suggesting that mutation in this gene has an effect similar to that of BRCA1 and 2 mutations.

It should also be underlined that there were also tumours with mutations in other HR genes classified as $\mathrm{LOH}$ low and which did not respond. So, it could be hypothesized that not all genes within the HR pathway are equivalent and therefore just relying on panel sequencing might not be sufficient.

Confirmed RECIST responses were more frequent in the LOH high subgroup (24 of 82 patients [29\%; 95\% CI 20-40]) than in the LOH low subgroup (7 of 70 patients [10\%; 95\% CI 4-20; $\mathrm{p}=0.0033]$ ), and the duration of response was longer in the LOH high subgroup (10.8 months; 95\% CI 5.7-not reached) than in the LOH low subgroup (5.6 months; $95 \%$ CI 4.6-8.5; $\mathrm{p}=0.022$ ).

A recent presentation compared efficacy results between the pre-specified genomic $\mathrm{LOH}(14 \%)$ and a 'refined' LOH cut-off point (16\%) and demonstrated an improvement in the selection of patients most likely to benefit from rucaparib (ORR of $80 \%$ in patients with $B R C A$ mutations for both LOH cut-offs but an improvement in ORR 
from 35 to $39 \%$ in patients with a $B R C A$-like LOH-high signature and from 13 to $14 \%$ in patients without a $B R C A$ mutation or a $B R C A$-like low signature) [39].

The HRD assay is also being prospectively tested in an extension (Part 2) of ARIEL2, in which rucaparib is administered in patients with platinum-sensitive, platinum-resistant, or platinum refractory cancers, who have received at least three but not more than four prior lines of therapy and have had a treatment-free interval of more than 6 months following first-line chemotherapy. The primary endpoint of ARIEL2 Part 2 is response rate. More importantly, the HRD test is also being applied prospectively in ARIEL3 (NCT01968213), a study of maintenance rucaparib in women with relapsed platinum-sensitive HGSC. In addition, a phase III study, ARIEL4 (NCT02855944), is ongoing, investigating rucaparib versus chemotherapy in patients with relapsed platinum-sensitive $g B R C A m$-only ovarian cancers who have received at least two lines of chemotherapy.

In December 2016, based on the positive results of the ARIEL clinical trials and other rucaparib studies, the FDA approved rucaparib as treatment for women with advanced ovarian cancer who have already been treated with at least two chemotherapies and have a $B R C A 1$ or $B R C A 2$ mutation identified by an approved companion diagnostic test [40].

\section{References}

1. Cancer Genome Atlas Network. Integrated genomic analyses of ovarian carcinoma. Nature 2011;474(7353):609-15.

2. Mukhopadhyay A, Elattar A, Cerbinskaite A et al. Development of a functional assay for homologous recombination status in primary cultures of epithelial ovarian tumor and correlation with sensitivity to poly(ADP-ribose) polymerase inhibitors. Clin Cancer Res 2010;16(8):2344-51.

3. Bryant HE, Petermann E, Schultz N et al. PARP is activated at stalled forks to mediate Mre11-dependent replication restart and recombination. EMBO J 2009;28(17):2601-15.

4. Farmer H, McCabe N, Lord CJ et al. Targeting the DNA repair defect in BRCA mutant cells as a therapeutic strategy. Nature 2005;434(7035):917-21.

5. Tan TZ, Miow QH, Huang RY et al. Functional genomics identifies five distinct molecular subtypes with clinical relevance and pathways for growth control in epithelial ovarian cancer. EMBO Mol Med 2013;5(7):1051-66.

6. Ahmed AA, Etemadmoghadam D, Temple J et al. Driver mutations in TP53 are ubiquitous in high grade serous carcinoma of the ovary. J Pathol 2010;221(1):49-56.

7. Pennington KP, Walsh T, Harrell MI et al. Germline and somatic mutations in homologous recombination genes predict platinum response and survival in ovarian, fallopian tube, and peritoneal carcinomas. Clin Cancer Res 2014;20(3):764-75.

\section{Conclusions}

Overall, response to PARPi seems to be strongly related to defective HR: broadly, those patients who present with a defect of function of the genes linked with HR pathways should achieve the greater benefit from PARP inhibition. This approximation holds for germline and somatic mutation in BRCA1 or BRCA2 and possibly RAD51C, but is not perfectly confirmed when HR is assessed using panel sequencing alone. HRD assays, based upon genomic $\mathrm{LOH}$ and other measures of genomic instability, show great promise, especially when added to $B R C A 1 / 2$ mutation state, but further refinement and validation is required for routine clinical use. In the maintenance setting, response to platinum chemotherapy, as demonstrated for both olaparib and niraparib, remains a powerful and useful phenotypic predictor of HRD and, therefore, of potential PARP sensitivity.

\section{Acknowledgments}

The authors thank Ray Hill, an independent medical writer, who provided native English editing and journal styling on behalf of HPS. This editorial assistance was funded by PharmaMar, Spain.

\section{Conflicts of Interest}

The Authors declare there are no conflicts of interest in relation to this article.

8. Hughes-Davies L, Huntsman D, Ruas M et al. EMSY links the BRCA2 pathway to sporadic breast and ovarian cancer. Cell 2003;115(5):523-35.

9. Bajrami I, Frankum JR, Konde A et al. Genome-wide profiling of genetic synthetic lethality identifies CDK12 as a novel determinant of PARP1/2 inhibitor sensitivity. Cancer Res 2014;74(1):287-97.

10. Konstantinopoulos PA, Ceccaldi R, Shapiro GI et al. Homologous recombination deficiency: Exploiting the fundamental vulnerability of ovarian cancer. Cancer Discov 2015;5(11):1137-54.

11. Ceccaldi R, O'Connor KW, Mouw KW et al. A unique subset of epithelial ovarian cancers with platinum sensitivity and PARP inhibitor resistance. Cancer Res 2015;75(4):628-34.

12. van den Bosch M, Lohman PH, Pastink A. DNA doublestrand break repair by homologous recombination. Biol Chem 2002;383(6):873-92.

13. McVey M, Lee SE. MMEJ repair of double-strand breaks (director's cut): deleted sequences and alternative endings. Trends Genet 2008;24(11):529-38.

14. Arnaudeau C, Lundin C, Helleday T. DNA double-strand breaks associated with replication forks are predominantly repaired by homologous recombination involving an exchange mechanism in mammalian cells. J Mol Biol 2001;307(5):1235-45. 
15. Walsh CS. Two decades beyond BRCA1/2: Homologous recombination, hereditary cancer risk and a target for ovarian cancer therapy. Gynecol Oncol 2015;137(2):343-50.

16. Fong PC, Boss DS, Yap TA et al. Inhibition of poly(ADPribose) polymerase in tumors from BRCA mutation carriers. N Engl J Med 2009;361(2):123-34.

17. Mirza MR, Monk BJ, Herrstedt J et al. Niraparib maintenance therapy in platinum-sensitive, recurrent ovarian cancer. N Engl J Med 2016;375(22):2154-64.

18. Swisher EM, Lin KK, Oza AM et al. Rucaparib in relapsed, platinum-sensitive high-grade ovarian carcinoma (ARIEL2 Part 1): an international, multicentre, open-label, phase 2 trial. Lancet Oncol 2017;18(1):75-87.

19. Marquard AM, Eklund AC, Joshi T et al. Pan-cancer analysis of genomic scar signatures associated with homologous recombination deficiency suggests novel indications for existing cancer drugs. Biomark Res 2015;3:9.

20. Pierce AJ, Johnson RD, Thompson LH et al. XRCC3 promotes homology-directed repair of DNA damage in mammalian cells. Genes Dev 1999;13(20):2633-8.

21. Fong PC, Yap TA, Boss DS et al. Poly(ADP)-ribose polymerase inhibition: frequent durable responses in BRCA carrier ovarian cancer correlating with platinum-free interval. J Clin Oncol 2010;28(15):2512-9.

22. Ledermann J, Harter P, Gourley C et al. Olaparib maintenance therapy in patients with platinum-sensitive relapsed serous ovarian cancer: a preplanned retrospective analysis of outcomes by BRCA status in a randomised phase 2 trial. Lancet Oncol 2014;15(8):852-61.

23. Gelmon KA, Tischkowitz M, Mackay $\mathrm{H}$ et al. Olaparib in patients with recurrent high-grade serous or poorly differentiated ovarian carcinoma or triple-negative breast cancer: a phase 2, multicentre, open-label, non-randomised study. Lancet Oncol 2011;12(9):852-61.

24. Campbell PJ, Stephens PJ, Pleasance ED et al. Identification of somatically acquired rearrangements in cancer using genome-wide massively parallel paired-end sequencing. Nat Genet 2008;40(6):722-9.

25. Geigl JB, Obenauf AC, Schwarzbraun T et al. Defining 'chromosomal instability'. Trends Genet 2008;24(2):64-9.

26. Abkevich V, Timms KM, Hennessy BT et al. Patterns of genomic loss of heterozygosity predict homologous recombination repair defects in epithelial ovarian cancer. $\mathrm{Br}$ J Cancer 2012;107(10):1776-82.

27. Birkbak NJ, Wang ZC, Kim JY et al. Telomeric allelic imbalance indicates defective DNA repair and sensitivity to DNA-damaging agents. Cancer Discov 2012;2(4):366-75.

28. Popova T, Manie E, Rieunier G et al. Ploidy and largescale genomic instability consistently identify basal-like breast carcinomas with BRCA1/2 inactivation. Cancer Res 2012;72(21):5454-62.
29. Timms KM, Abkevich V, Neff C et al. Association between BRCA1/2 status and DNA-based assays for homologous recombination deficiency in breast cancer [Abstract]. Thirty-Sixth Annual CTRC-AACR San Antonio Breast Cancer Symposium Dec 10-14 2013, San Antonio, TX, USA. Cancer Res 2013;73(24 suppl):Abstract P6-05-10.

30. Telli ML, Timms KM, Reid J et al. Homologous recombination deficiency (HRD) score predicts response to platinum-containing neoadjuvant chemotherapy in patients with triple-negative breast cancer. Clin Cancer Res 2016;22(15):3764-73.

31. Sakai W, Swisher EM, Karlan BY et al. Secondary mutations as a mechanism of cisplatin resistance in BRCA2mutated cancers. Nature 2008;451(7182):1116-20.

32. Norquist B, Wurz KA, Pennil CC et al. Secondary somatic mutations restoring BRCA1/2 predict chemotherapy resistance in hereditary ovarian carcinomas. J Clin Oncol 2011;29(22):3008-15.

33. Konstantinopoulos PA, Spentzos D, Karlan BY et al. Gene expression profile of BRCAness that correlates with responsiveness to chemotherapy and with outcome in patients with epithelial ovarian cancer. J Clin Oncol 2010;28(22):3555-61.

34. Davies H, Glodzik D, Morganella S et al. HRDetect is a predictor of BRCA1 and BRCA2 deficiency based on mutational signatures. Nat Med 2017;23(4):517-25.

35. Shen Y, Aoyagi-Scharber M, Wang B. Trapping poly(ADPribose) polymerase. J Pharmacol Exp Ther 2015;353(3): 446-57.

36. Konecny GE, Kristeleit RS. PARP inhibitors for BRCA1/2mutated and sporadic ovarian cancer: current practice and future directions. Br J Cancer 2016;115(10):1157-73.

37. Sandhu SK, Schelman WR, Wilding G et al. The poly(ADPribose) polymerase inhibitor niraparib (MK4827) in BRCA mutation carriers and patients with sporadic cancer: a phase 1 dose-escalation trial. Lancet Oncol 2013;14(9):882-92.

38. US Food and Drug Administration (FDA). FDA approves maintenance treatment for recurrent epithelial ovarian, fallopian tube or primary peritoneal cancers. 27 March 2017. Available from: https://www.fda.gov/NewsEvents/Newsroom/PressAnnouncements/ucm548948.htm.

39. Coleman R, Swisher E, Oza A et al. Refinement of prespecified cutoff for genomic loss of heterozygosity $(\mathrm{LOH})$ in ARIEL2 part 1: A phase II study of rucaparib in patients (pts) with high grade ovarian carcinoma (HGOC) [Abstract]. J Clin Oncol 2016;34(suppl).

40. US Food and Drug Administration (FDA). FDA grants accelerated approval to new treatment for advanced ovarian cancer [media release]. 19 Dec 2016. Available from: https://www.fda.gov/newsevents/newsroom/pressannouncements/ucm533873.htm. 